\title{
Overview of Highly Flexible, Deployable Lattice Structures Used in Architecture and Civil Engineering Undergoing Large DisPLACEMENTS
}

\author{
Noemi Friedman ${ }^{1,2}$, Adnan Ibrahimbegovic ${ }^{3}$ \\ ${ }^{1}$ Institute of Civil Engineering, \\ Ybl Miklós Faculty of Architecture and Civil Engineering, Szent István University, Budapest, Hungary \\ ${ }^{2}$ Institute of Scientific Computing, TU Braunschweig, Germany \\ n.friedman@tu-braunschweig.de \\ ${ }^{3}$ Laboratory of Mechanics and Technology, Ecole Normale Supérieure de Cachan, France \\ adnan.ibrahimbegovic@lmt.ens-cachan.fr
}

\begin{abstract}
In this article an extensive but not exhaustive review on different transformable systems - retractable roofs, deployable and retractable pantographic lattice systems, pop-up and snap-through type structures, tensegrity structures - used in architecture and civil engineering will be given. This study was carried out to explore earlier and current researches and technologies to demonstrate the available systems and their potential. However, the main goal of this article is to expose the wild range of structural systems requiring elaborate dynamical analysis, divers of them are still lacking profound calculation procedure.

The reviewed systems are herein categorized in two groups: 1) multibody systems having additional controlling and stabilizing elements and 2) systems undergoing large deformation, and instability phenomena. The article will pan out about the applicable joint types, some of them involving friction and damping effects. For a better outline, simplified analysis of elementary segments of some reviewed systems is annexed.
\end{abstract}

Keywords: deployable structures, transformable systems, dynamic architecture, pop-up systems, snapthrough structures

\section{INTRODUCTION}

Corresponding to new concepts of the 21 th century and due to available numerical and robotics technologies advanced transformable structures have appeared in civil engineering and architecture [e.g.1-5]. Structures used for off-shore industry, light deployable structures used for provisory and transformable architecture can be mentioned among these shape morphing 
structures that can change shape like the wing of the insects or the petals of the flowers or like the movable structure of human body. In this article we restrict our attention only to lattice deployable structures.

These structures are designed to undergo very large displacements and remain fully operational [6]. Often structures of this kind can integrate a multibody system (e.g. [7] or [8]) which facilitates a construction phase before being integrated in a structural assembly (e.g. [9]). Modeling of the component of 3D frame-type flexible structures of this type is nowadays under control - thanks to the geometrically exact beam model [10-11] capable of representing large displacements and rotations, and solving the pertinent instability problems [12].

However, not enough is known about the dynamics of the highly flexible structures of this kind. The dynamical analysis of these structures addresses problems like vibration control, the study of the true nature of damping and its proper numerical implementation, the development of the time-integration schemes which are suitable for handling different deformation modes with potentially large difference in associated stiffness and natural frequencies - or what is referred to the stiff differential equations.

The main goal of this article is not to address these problems but rather to expose the wild range of structural systems to the Reader being expert in the necessary elaborated dynamical analysis. This is important due to the lack of profound calculation procedure for varaibles of the reviewed systems.

In this article deployable structures are classified into two groups: 1) multibody systems, mechanisms that are controlled and stabilized by additional elements and 2) highly flexible structures undergoing large displacements, deformation and instability phenomena.

The former impose the problem of finding fast and easy control to obtain the needed geometrical configurations while the stiffness of the structure has to be also assured in the operational configurations. In some cases this implies only two separated configurations; the initial, compact configuration which allows the structure to be stored and transported securely, and the deployed final configuration that has to resist to the operational loads. To assure the operational stiffness of the structure additional stabilizing elements can be added after the deployment. In the case of an adaptive structure the aim is rather to assure the required stiffness of the structure in infinite number of configurations, that is the structure can continuously change its morphological properties while staying fully operational. Though significantly increasing the complexity of the system, the application of continuously changing morphological structures prospects a dynamical architecture that can not only change its properties with the aim to ameliorate occupant comfort and to adapt the structure to changing occupant demands but also for reducing environmental impact and for a better energy efficiency.

Using structures that are packed by imposing large elastic deformations and by involving instability phenomenon either self-stabilizing or self-deploying (pop-up) systems can be created. These structures can serve for a fast constructional method that has extreme importance in airspace structures but can serve well for provisory architecture and military installations, as well.

The force-displacement diagrams of the basic segments of self-stabilizing and self-deploying systems are similar in respect of having zero packing/deploying force, that corresponds to both the deployed and the packed configurations. In between these two configurations the structure has to pass a critical state corresponding to the maximal value of the packing/deploying force. After this critical state further smooth displacement can be only achieved by descending force. 
Consequently, if force control is applied, then the phenomena will cause an instable phase, causing a sudden displacement, that is, a snapping of the structure. Smooth transformation process can only be realized by displacement control.

Despite the similar characteristic of the equilibrium paths there is a major difference between self-locking and self-deploying structures. For self-locking pantographic system the deployed and the packed configurations correspond to a stable, strain-free state due to the special geometric configuration resulting in a second stable phase (after reaching the minimum value of the packing force) of the equilibrium path. On the contrary, the packed configuration of the pop-up systems is characterized by an unstable state corresponding to the maximal value of the cumulated strain-energy. In this compact configuration the elements are not stress- and strainfree and due to the instability of the state a small perturbation results in a sudden snapping of the structure to the initial, stress-free configuration. This characteristic is herein demonstrated though force-displacement diagrams of the basic segment of such structures.

Due to the sudden snapping during packing/deploying the inertial effects cannot be neglected and consequently a profound dynamic deployment and packing analysis is required for the analysis of such structures. In this article the applicable joint construction will be also outlined, which can often involve friction and damping effects. In the following, the topology of the different systems will be demonstrated by throughly presenting some elementary segments of the system which makes its mechanical behavior clear by some simplified analysis.

\section{STABILIZED AND CONTROLLED MULTIBODY SYSTEMS}

\subsection{Multibody systems for the erection of the structure}

\section{Deployable Tensegrity Structures}

The experimental system, called tensegrity, was born at the end of the 1940s from the artistic exploration of K. Snelson and Fuller's goal of creating maximal efficiency structures [13]. Snelson called his tensegrity sculptures the "floating compression" system, while the word "tensegrity" (from tensile integrity) is coined by Fuller. This spatial truss system's elements can be separated to purely compressed and purely tensile components. With this separation the tensioned members can be as light weight as current material technology allows, resulting extremely light, economical and less visually intrusive structures [14].

a)

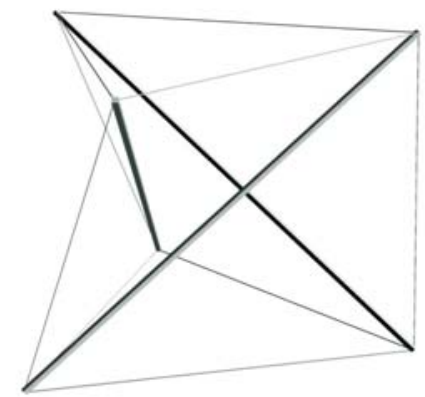

b)

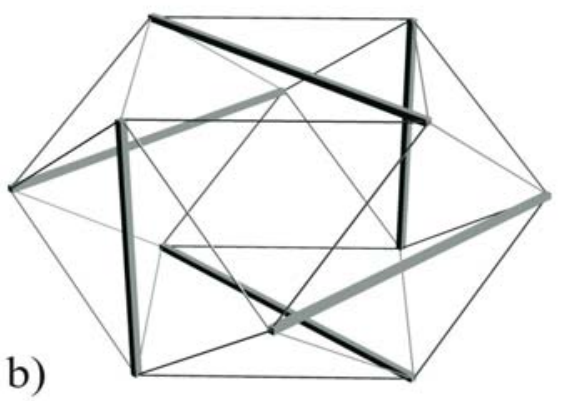

Figure 1: Examples for tensegrity units: a) the tensegrity tripod b) expanded octahedron 
The simplest tensegrity unit is the tensegrity tripod [15] (Fig. 1a) and other tensegrity networks can be derived from geodesic polyhedra [16] (Fig. 1b). Assembling these units planar and spherical structures can be created, therefore they can be used for walls, floors and roofs or bridges.

The idea to have only tendons connected to struts is a very innovative concept of this type of structures. It results extremely simple joints, but difficulties are imposed when trying to connect these exotic structures with conventional architectural structures as e.g. cladding in the case of a dome.

Though tensegrities have pre-stressed mechanisms in their static configuration, this mechanisms are only infinitesimal, thus the classical tensegrities are not deployable systems. However, derived from the intrinsic property of tensegrity structures foldability can be easily obtained by changing the lengths of the elements of the structures. This can be either the changing of strut length by using telescopic bars or the folding can be enriched by changing the length of the cable. The main difficulty of the former method is that in the folded configuration the cable often creates an inextricable tangle opposing thus the system to unfold [17]. Recently innovative deployable structures were born by the application of the basic ideas of the tensegrities, as e.g. the mobile bridge design developed for a fictitious journey on Mars by the Department of Structural Design of the TU Munich [18] or the deployable space truss system being also a foldable bridge realization, proposed by Rhode-Barbarigos et al. [19].

\section{Pantographic structures folding into a bundle}

a)
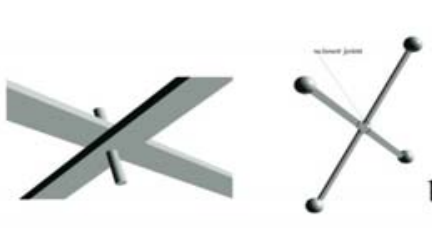

b)

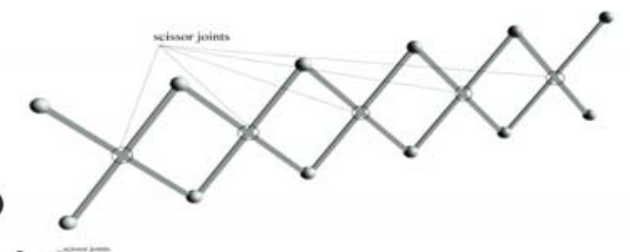

c)

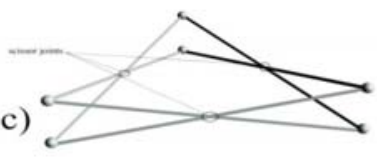

d)

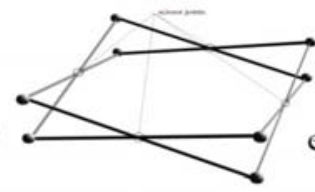

e)

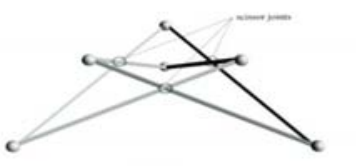

g)

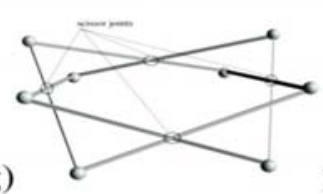

f)

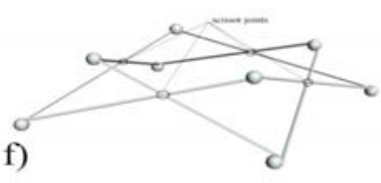

i)
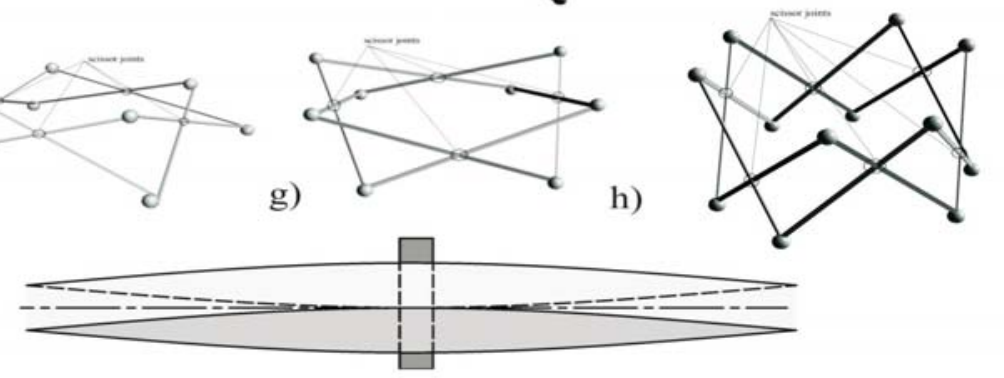

Figure 2 A simple scissor joint and the basic element, one SLE (a); the lazy tong (b) and some secondary basic units of scissor-like deployable structures (basic units with symmetrical scissors (c, d), pyramid type units (e, f) skew type units $(\mathrm{g}, \mathrm{h})$ ); bent scissor elements (i) 
A large number of structures that can be opened and closed, are based on the well-known concept of the lazy tong system. The minimum component of this system is the so-called scissor like element (SLE henceforth). The SLE consists of two bars connected to each other with a revolute joint (Fig. 2a). By the parallel connection of SLEs the simplest 2D deployable structure, the lazy tong is constructed (Fig. 2b). Connecting at least three of SLEs through complete pin joints a ring is formed, providing a secondary unit of this frame structure (Fig. 2c-h). The scissors can be folded to bundle if the foldability constraint is fulfilled:

$$
\mathrm{a}+\mathrm{b}=\mathrm{c}+\mathrm{d}
$$

Where a, b, c, d are the lengths of scissor parts measured from the revolute joint of the adjacent scissors (Fig. 2a).

When connecting SLEs to a ring, the joints at the four endpoints of the scissors have to stay in the same plane. Consequently if the simplest, asymmetrical cylindrical joint is applied (Fig. 2a) the scissors had to be bent (Fig. 2i) because of the extension of the rods (in order to keep their four endpoint in the same plane). This results in the occurrence of transverse forces in the joints resulting in friction when trying to rotate them [1].

By the further connection of secondary units almost all kind of 3D shapes can be formed folding into bundle. Adding tension components like wire or membrane to its developed form, it becomes a 3D truss and gets effective strength. With this system towers, bridges, domes (e.g. Figs. 3.) and space structures can be rapidly constructed [20].

a)

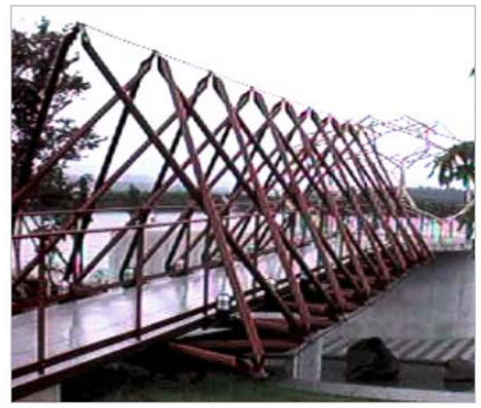

b)

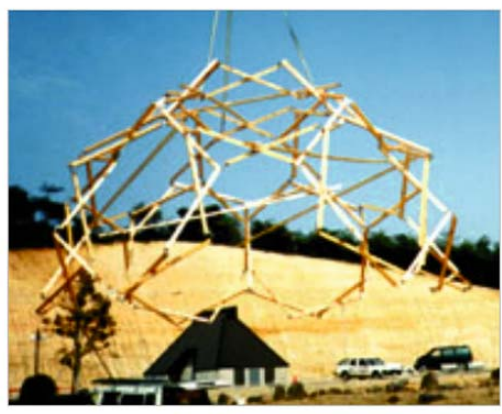

c)

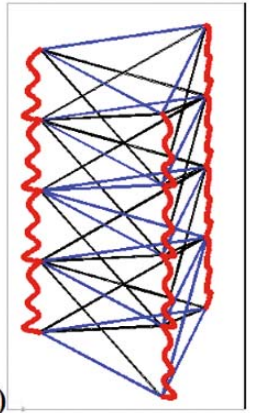

Figure 3 Foldable bridge (a) and deployable dome (b) by ATAKE Space Design Lab. Co. in the Hanamizuki Park, Japan '96 [20]; schematic drawing of the deployable mast controlled by active and passive cables by the former Deployable Structures Laboratory (c)

(passive cables with red lines, active cables with blue lines, scissors with black)

Using scissor-like deployable structures in architecture was pioneered by the Spanish engineer, E. P. Piñero. He presented a foldable theatre in 1961 [21], and elaborated several other deployable designs. The biggest drawbacks of his designs were the relatively heavy and big joints due to eccentric connections and necessary temporary support as the structure was stiffened by intermediate bars or tension elements that were added after the structure was deployed into the desired configuration [1]. Despite of all the disadvantages of his structures, Piñero inspired several researchers. 
This was the case with Professor F. Escrig, who designed the $30 \mathrm{~m} \times 60 \mathrm{~m}$ deployable roof for a swimming pool in Seville [22, 23]. By piling up symmetrical or pyramid type structural units (Fig. 2e-f) vertically a basic pantographic structure is formed: a three-dimensional mast [20]. The only internal degree of freedom of the deployable mast developed in the former Deployable Structures Laboratory is controlled by a single, continuous "active" cable (Fig. 3c) which runs over pulleys connected to the joints of the pantograph [24]. A series of short (initially loose) cables linking neighboring joints of the pantograph become taut when the pantograph is fully deployed, and in this configuration, the continuous cable imparts a global state of prestress onto the whole structure. The application of this vertical connection horizontally results a foldable bridge, which is useful for its rapid construction [20]. An example is realized in the Hanamizuki Park (Fig. 3a).

\section{Pantadome erection}

3D spatial structures are extremely efficient ones completed. However the difficulties with installation (big amount of scaffolding, labor and time) often highly decrease this efficiency. This drawback can be significantly reduced with the unique structural pantographic system called the Pantadome System invented by M. Kawaguchi and will be herein explained in accordance with [25].

The principle of this structural system is to make a dome or a conical space frame unstable for a period of the construction so that it is "foldable" during its erection. This can be done by temporarily taking out the members lying on a hoop circle (Fig. 4) then the dome is given a "mechanism", like a 3D version of a parallel crank or a "pantograph".

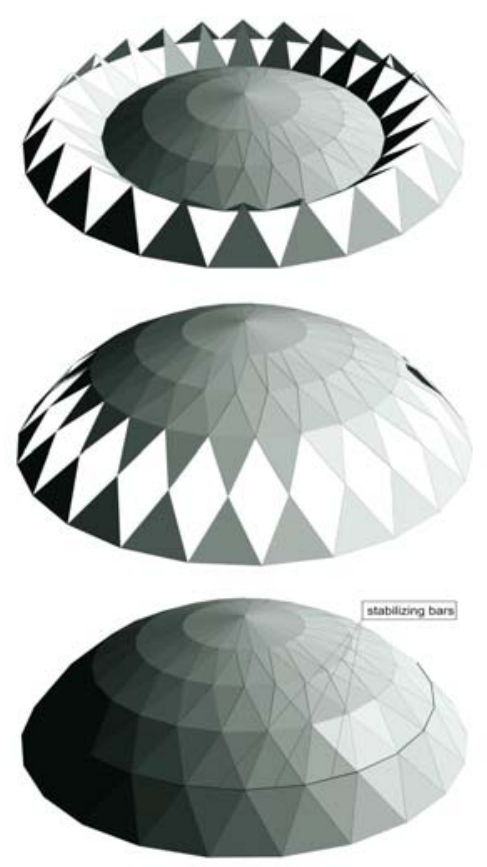

Figure 4 Schema of the of the pantadome erection 
Since such a dome is assembled in a folded shape near the ground level and the entire height of the dome during assembly work is very low compared with that of after completion, the assembly work can be done safely and economically, and the quality of work can be assured more easily than in conventional erection systems. Not only the structural frame but also the exterior and interior finishings, electricity and mechanical facilities can be fixed and installed at this stage. The dome is then lifted up. Lifting can be achieved either by blowing inside the dome to raise the internal air pressure or by pushing up the periphery of the upper dome by means of hydraulic jacks. The major advantage of this system compared with different lifting solutions is that no guying cables or bracing elements are necessary for lateral stability. This is due to the fact that the mechanism of the system can be controlled with only freedom of movement in the vertical direction. When the dome has taken the final shape, the hoop members which have been temporarily taken away during the erection are fixed to their proper positions to complete the dome structure. Several designs have been realized in accordance to the pantadome principle. One is the Namihaya Dome with diameter of $127 \mathrm{~m}$ and $111 \mathrm{~m}$.

\section{Other cable and strut systems, mixed systems}

For architectural and special applications several deployable lattice systems were invented using ideas differing from the already mentioned pantographic or tensegrity systems and some use a mixture of the mentioned types. Herein only a few examples will be presented without scoping an exhaustive list.
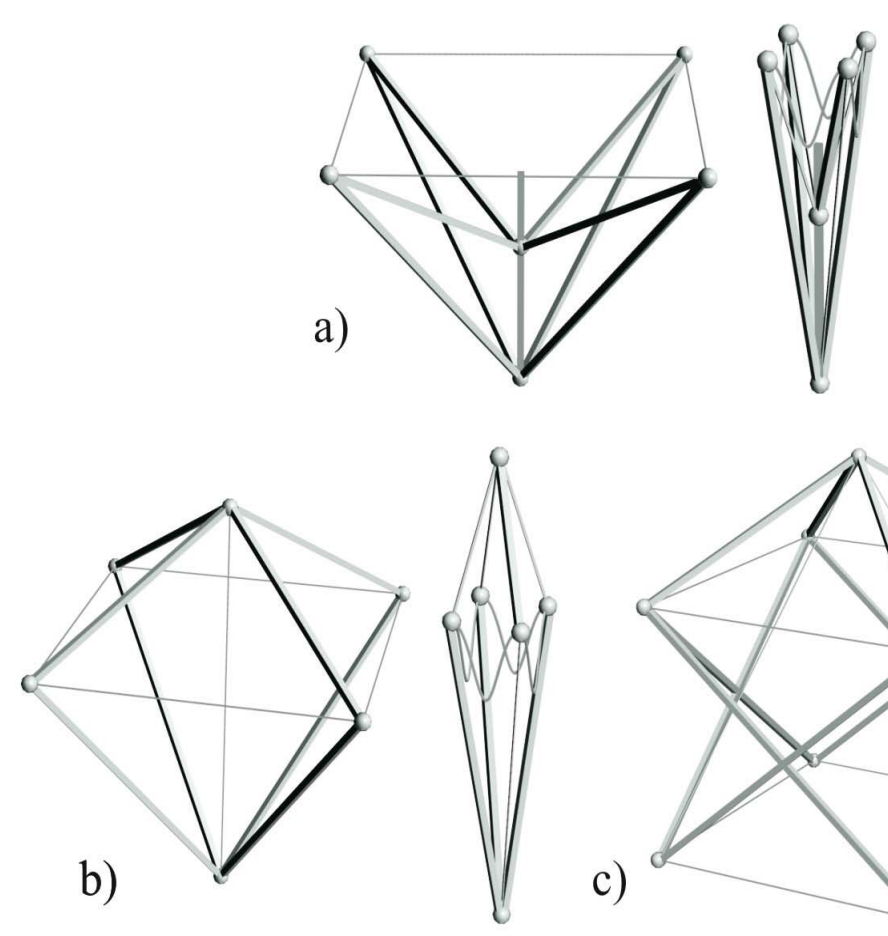

c)
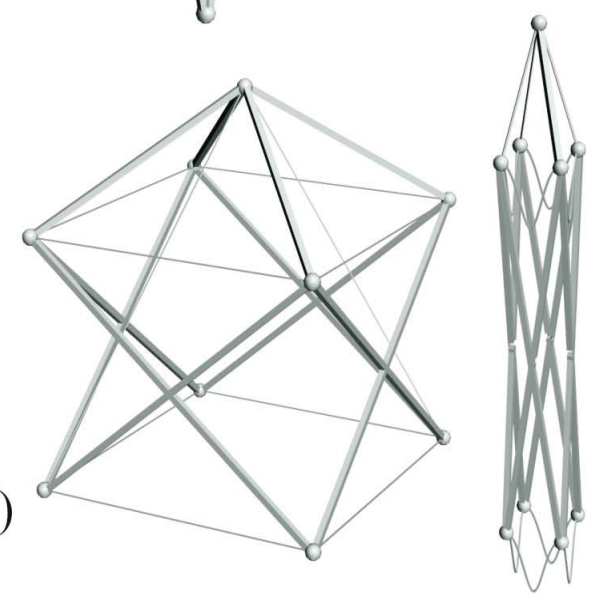

Figure 5 Post-stabilized cable-strut deployable structural units by Liew and Krishnapillai 
Some foldable lattice designs use sliding joints for transformation. This is the case with the ancient structure of the umbrella. Sliding joint is used for the 3D foldable "hexapod" of Onoda et al. [26] or the pyramid type cable strut systems of Liew and Krishnapillai et al. [27, 28] (Fig. 5a). Liew and Krishnapillai introduced a new family of deployable units combining cable-strut systems with foldability features similar to the deployable tensegrity structures. The basic element of these structures use, either sliding joints (Fig. 5a) or cables stiffening (Fig. $5 b-c)$ based on the 'deploy \& stabilize' method. This system is rather applicable for terrestrial use as elements need post-stabilizing and consequently with these units structures cannot be instantly deployed, the on-site assembling is more laborious. These structures were thoroughly analyzed by Vu et al. [4]. Due to their paper the novel system proves a remarkable structural efficiency (calculating from the optimized structure's total load/structural self-weight and the span/deflection ratios) that is even comparable with conventional nondeployable double layer lattice designs and it is also fast and easy to construct (compared with conventional double layer structures) while the weight of the structures is still competitive. For an example of architectural application of cable pantographic elements a $30 \mathrm{~m}$ spanning deployable membrane structure supported by two deployable inclined arches was offered by Tran et al. [29].

\subsection{Multibody systems for continuous transformability}

\section{Pantographic structures}

Probably the simplest shape morphing structure is the pantographic arm or lazy tong presented in chapter 2.1. By adding active cables driven through the joints a pantographic arm can be constructed [1, pp. 131-133]. However the architectural application of pantographic structures that fold into a bundle are highly restricted.

C. Hoberman, the American engineer, made a considerable advance in the design of retractable roof structures by the discovery of the simple angulated element [30-31]. By the refraction of the two straight rods of a single SLE the angulated element is formed (Fig. 6). This element is able to open and close while maintaining the end nodes on radial lines that subtend a constant angle $[2,31,32]$.

a)

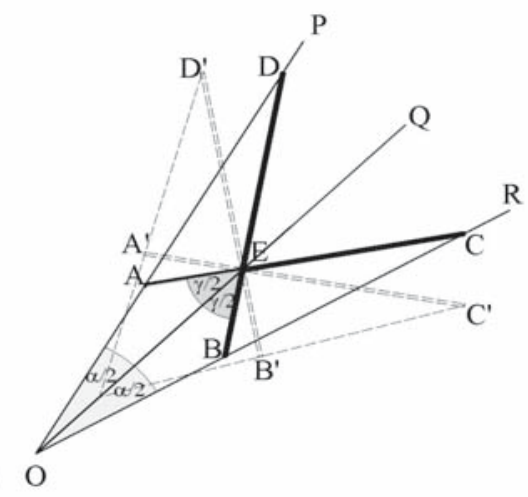

b)

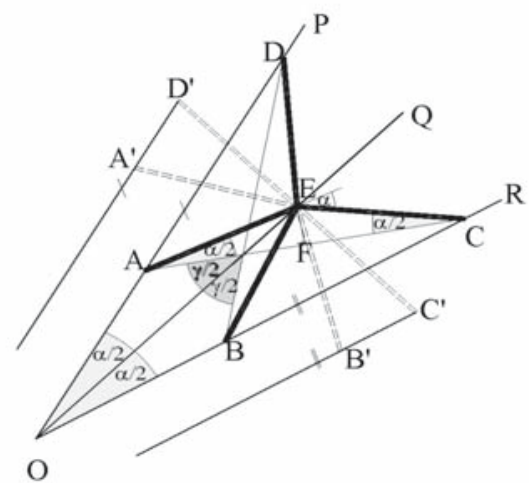

Figure 6 (a) Classic SLE and (b) the simple angulated element (illustrated in accordance with [32]) 
Using angulated elements Hoberman created the retractable $6.2 \mathrm{~m}$ tall and $10.2 \mathrm{~m}$ high Iris Dome, at the EXPO2000. The exhibition dome was formed by the connection of the angulated elements on concentric circles (Fig. 7). One of the drawbacks of this design is that the structure does not maintain a constant perimeter. On the other hand, for the construction of the relatively small span structure required more than 11400 machined pieces [33], which can cause potential problems with reliability and a laborious and expensive manufacturing. The complexity of the hinges is due to the special geometric configuration; as the hinge axes has to be perpendicular to the plane of the scissor, the interconnecting hinges are realized by a rigid body of small extension connected to four simple scissor hinges with four different hinge axis [34] (Fig. 7).

a)

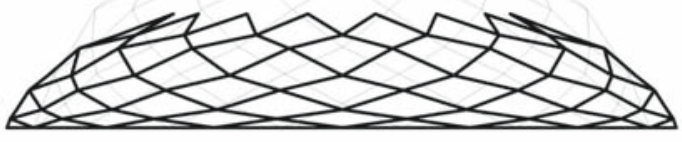

b)

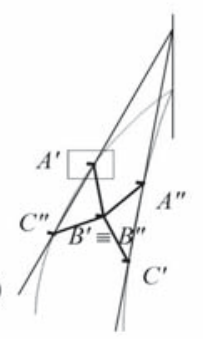

c)

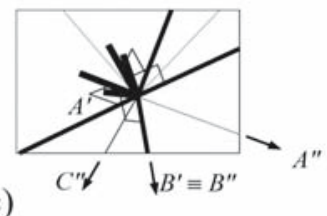

Figure 7 Iris dome designed by Hoberman : schematic model (a), complex hinge system (b, c) [35]

Further developments were made by Z. You and S. Pellegrino [32] by generalizing the angulated elements to a large family of foldable building blocks and by introducing a new type of pantographic structure based on the so called multi-angulated elements. Using multi-angulated elements the number and complexity of elements and joints of retractable trellis structures can be reduced. Each multi-angulated element is composed of a number of bars, which are rigidly connected to each other (Fig. 8), instead of consisting of separate angulated elements as used by Hoberman.
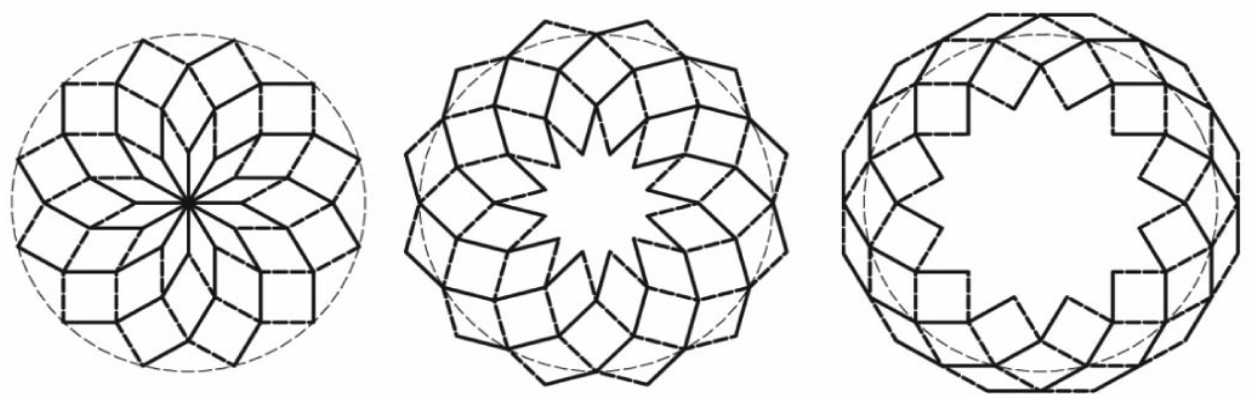

Figure 8: Deployable sequence of a ring structure developed by Z. You and S. Pellegrino 
The 3D version of the multi-angulated element can be generated by a vertical projection of all hinges to a hemisphere. The retractable structure elaborated at the former Deployable Structures Laboratory made from two layers of multi-angulated elements connected only by cylindrical joints with all hinge axis perpendicular to the plane. Though the projected retractable dome by You and Pellegrino have less complex joints, its opened configuration forms a less aesthetic toroidal-like shape that is more susceptible to wind effects [35].

Further developed and very aesthetic retractable dome system was tested by Kokawa [36], who identified a special geometry that enables all the joints to move on the sphere with scissor hinge axes pointing to the center point of the sphere. Unfortunately, in case of this configuration there is a slight difference in the direction of hinge axes and the hole axes during retraction, consequently either a loose hole or an embedded spherical roller bearing is needed [36].

All the above mentioned retractable dome models has the problem of variable perimeter radius. While Hoberman overcame this difficulty by supporting his Iris Dome with a wide annulus on which the domes joints on the edge can slide radially, the model of the Deployable Structures Laboratory and the one by Kokawa is supported with a secondary mechanism, a pin jointed support. P. E. Kassabian succeeded to change the geometry of the iris dome's structure by rigid body rotation, so that the motion of each angulated element is a pure rotation about a fixed point, and thus allows the application of fixed support points [37-38]. F. Kovács identified a new type of retractable iris dome combining simple scissor hinges and common hinges that has a fix outer ring, too [35].

\section{Other type of highly flexible lattice designs}

Practically by choosing the right actuation system all of the mechanism can be converted to shape morphing structures that are widely used in spatial structures and robotic technologies. With the help of ameliorating sensor systems and improving performance of light structural materials adaptive and intelligent architectural structures that can continuously change shape have got a foot in the door. The trend to design adaptive/responsive architectural applications turns the kinematic indeterminacy of tensegrity structures an advantage [39]. Deployable tensegrity targeting not only a packed and a deployed configuration but a wide variety of different shapes have been recently investigated. One example of adaptive roof design is the actuated tensegrity of A. Herder [40], who used computational design tools to develop a load bearing simple curved tensegrity shelter that is able to change shape, continuously.

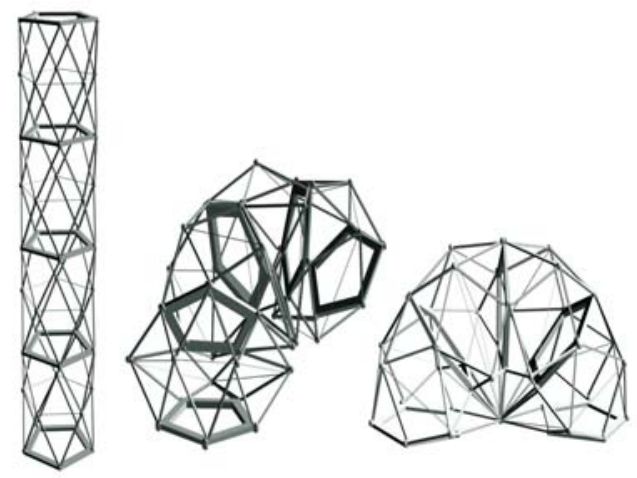

Figure 9 Shape morphing antiprismatic arm by the authors 
For other examples a tetrahedral truss system was presented by Sofla et al. [41] that can bend, twist and undulate by linear displacement actuators. A novel family of antiprismatic arm was investigated by the authors [5] (Fig. 9) derived from the yoshimura origami pattern. By polygonal actuators the arm can freely move in the space. The topology and geometry of the system initially used for a pop-up must was first proposed by Hegedüs [43] for a self-deploying mast (see later in Chapter 3.2).

\section{HIGHLY FLEXIBLE STRUCTURES UNDERGOING LARGE DISPLACEMENTS AND INSTABILITY PHENOMENA}

\subsection{Self-locking pantographic structures}
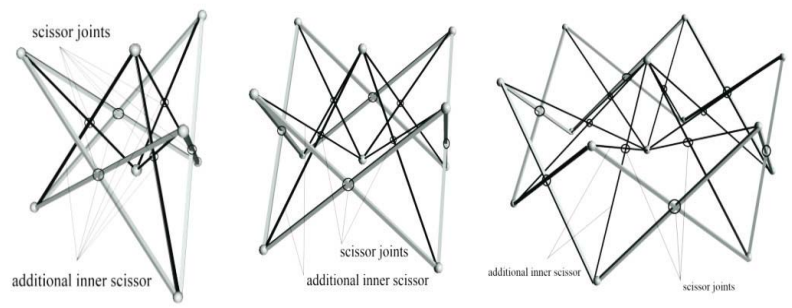

Figure 10 Self-stable deployable structures: secondary units for the planar self-locking structures

While pantograph structures, discussed above, all need additional stabilizing elements - like cables or other locking devices -, it is possible to design deployable structures that are selfstable in the erected configuration without any additional member with the application of a special geometric configuration [1].

This can be achieved by adding inner SLEs to the initial secondary units shown in Fig. 2. These units are shown in Fig. 10. The inner SLEs deform while unfolding due to geometric incompatibilities thus resulting a self-locking, self-stabilizing mechanism that locks the structure in its opened configuration [43] (cited by [1]). The first dome structure of this type was introduced by T. Zeigler in 1974 [44]. Several pop-up displays and pavilions are constructed in accordance with his patents.
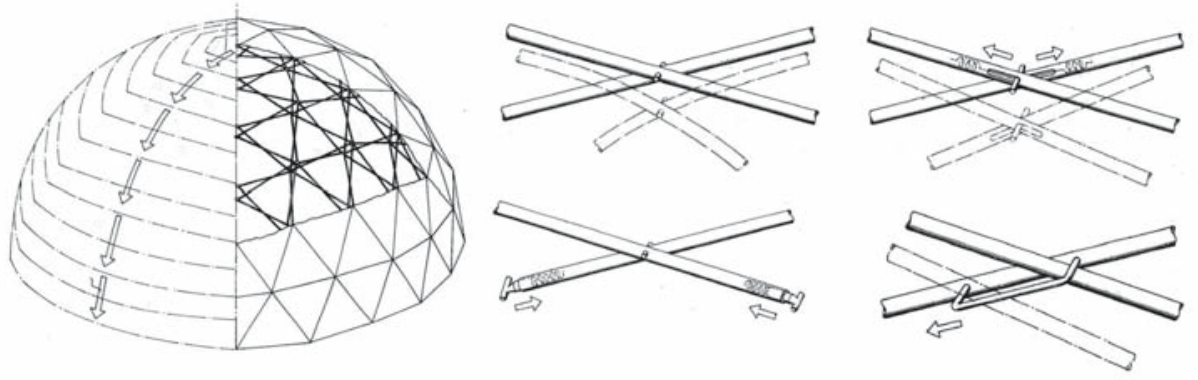

Figure 11 Zeigler's patent for collapsible self-supporting structure: dome and scissor details [45] 
Possible shapes with Zeigler's first pop-up dome patent [44] is strongly limited because of strict geometric restrictions. The major disadvantage of the structure though is that in the final configuration some members are not stress-free and remain curved. This residual stresses and bent form leads to a decrease of load bearing capacity and makes these structures more susceptible to catastrophic failures due to member buckling [1]. Zeigler improved his initial structure by introducing sliding joints for some intermediate nodes and flexible connections at the end nodes by springs [45] (Fig. 11)

For the elimination of both of the above mentioned problems - namely external stabilization and residual stresses in the deployed configuration - improved self-locking structures were investigated by A. Krishnapillai at the MIT [46-47] (cited by [1]). By the satisfaction of certain geometric constraints, these structures can be stable in the deployed configuration having straight and stress-free members (see Fig. 12b), except for dead weight and live load effects. During deployment, however, geometric incompatibilities result in the development of second order strains and stresses and a snap-through type of behavior that 'locks' the structure and assures its stability in the deployed configuration. About self-stable structures of this kind a practical and detailed design guide was published, written by Gantes [1], however, the published analysis exclude any dynamical investigation.

As an example the force-displacement diagram of a basic element of self-locking deployable structures are herein presented that can be used for planar assemblies. The outer SLEs would form a simple pantographic mechanism without the inner SLEs. The diagram was plotted from data gained from a numeric simulation (run by FEAP) with displacement control. The center bottom joint was fixed and the center top node was vertically displaced with "u" upwards until complete closure $(\mathrm{u}=\mathrm{h})$. It can be seen in Fig. 12 that no stresses occur in the outer SLEs (marked with o. SLE (1-4)), while during packing the inner SLEs (marked with i. SLE (5-8), see Fig. 12a) have to be deformed. Both, the deployed and the closed configurations correspond to zero force and a stress-free state.

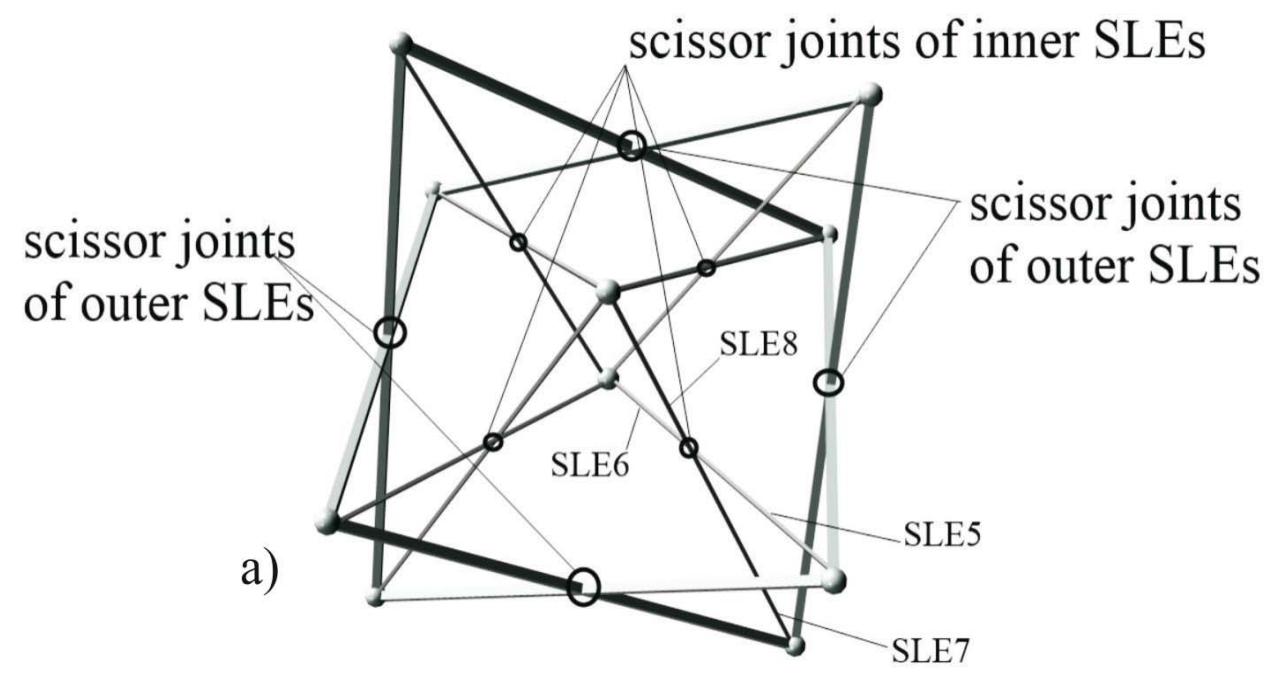




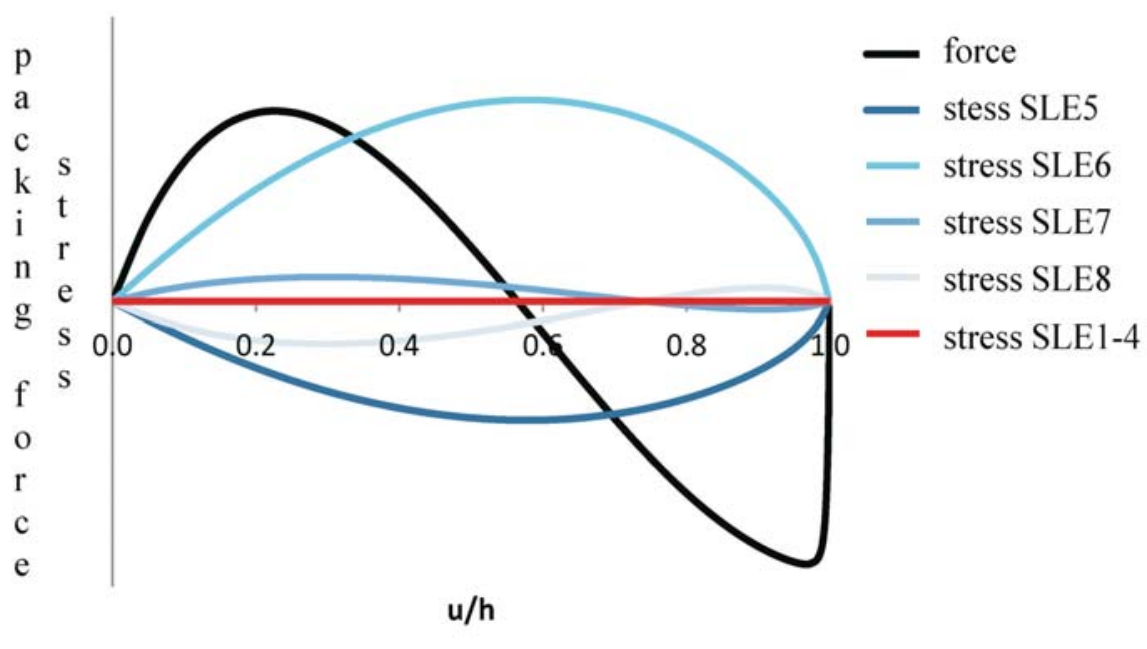

Figure 12 Packing simulation of a self-locking pantographic structure: basic simulated element (a); characteristic of the packing forces and the resulting stresses in the scissors in function of the relative displacement of the center node ( 0 corresponds to the deployed and 1 to the packed, planar state) (b)

\subsection{Self-deploying structures}

\section{Self-deployment with changing element-lengths}

Mikulas et al. presented possibilities of deploying planar trusses achieved by changing the length of the elements [48]. In contrast to self-locking structures (presented above), the packed configuration of these pop-up trusses is characterized by an unstable state corresponding to the maximal value of the cumulated strain-energy. In this compact configuration the elements are not stress- and strain-free and due to the instability of the state a small perturbation results in a sudden snapping of the structure to the initial, stress-free configuration. However, similarly to the self-locking structures, the deployment or the packing is achieved by deforming some elastic elements.

This behavior is herein presented through showing the behavior of the basic segment of the antiprismatic pop-up column proposed by Hegedüs [42] (Fig. 13). This column consists of rigid and elastic bars and rigid panels between the segments. The mast is packed by lengthening the horizontal elastic bars (Fig. 13).

The equilibrium equation is plotted in Fig. 14. The figure shows the downward packing force $(\mathrm{N})$ in function of the relative displacement (displacement of top nodes $(\mathrm{u}) /$ total height of the structure ( $2 \mathrm{~h})$. It can be seen that the force-displacement diagram of the structure is such that zero force corresponds to both, the deployed and the packed configuration [49]. While the former is a stable configuration, the latter is an unstable one. Consequently small locking devices are needed to stabilize and transport the structure in the packed configuration and the structure pops up when released. 

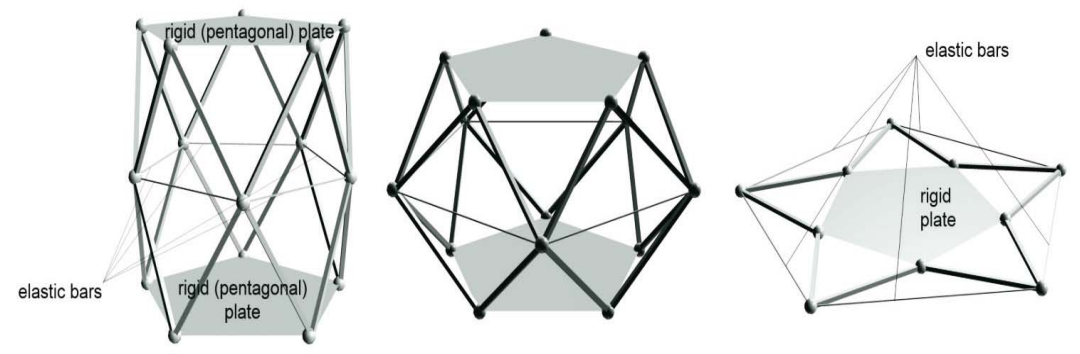

Figure 13 Basic segment and its packing sequent of antiprismatic pop-up mast proposed by Hegedüs

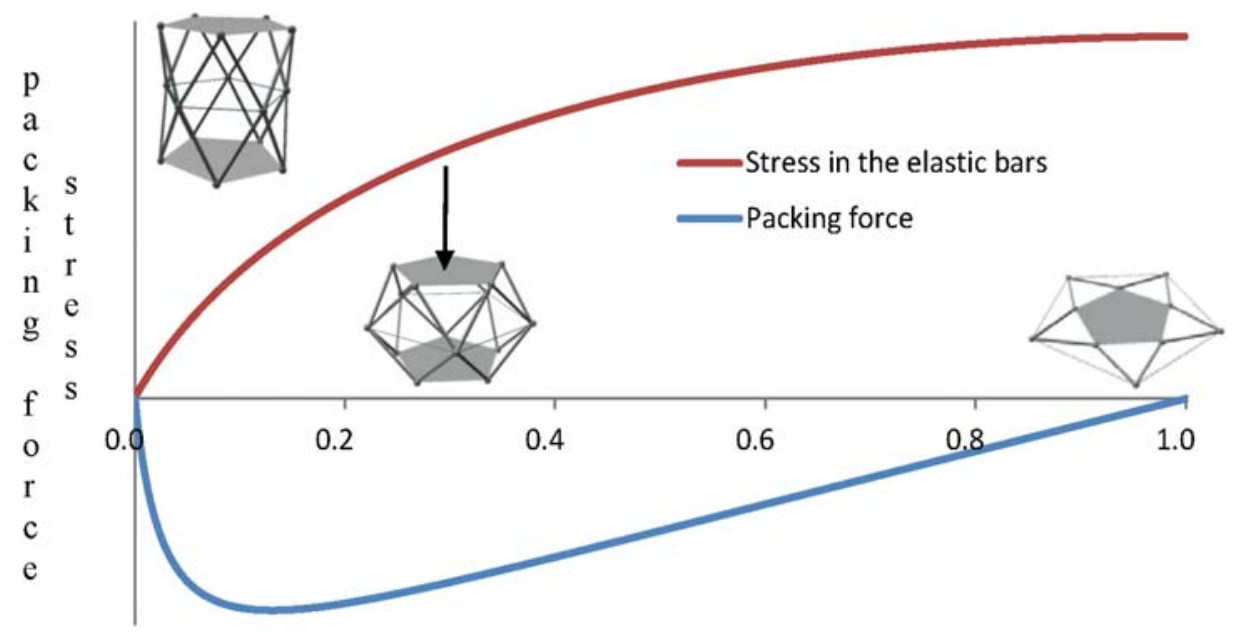

Figure 14 Packing (downward) force in function of the relative displacement of the top nodes and tensile stresses in the elastic, pentagonal bars ( 0 corresponds to the deployed and 1 to the packed, planar state)

The geometry of this pop-up mast could be used for small-span provisory pop-up bridges for example [5, 49] (Fig. 15).
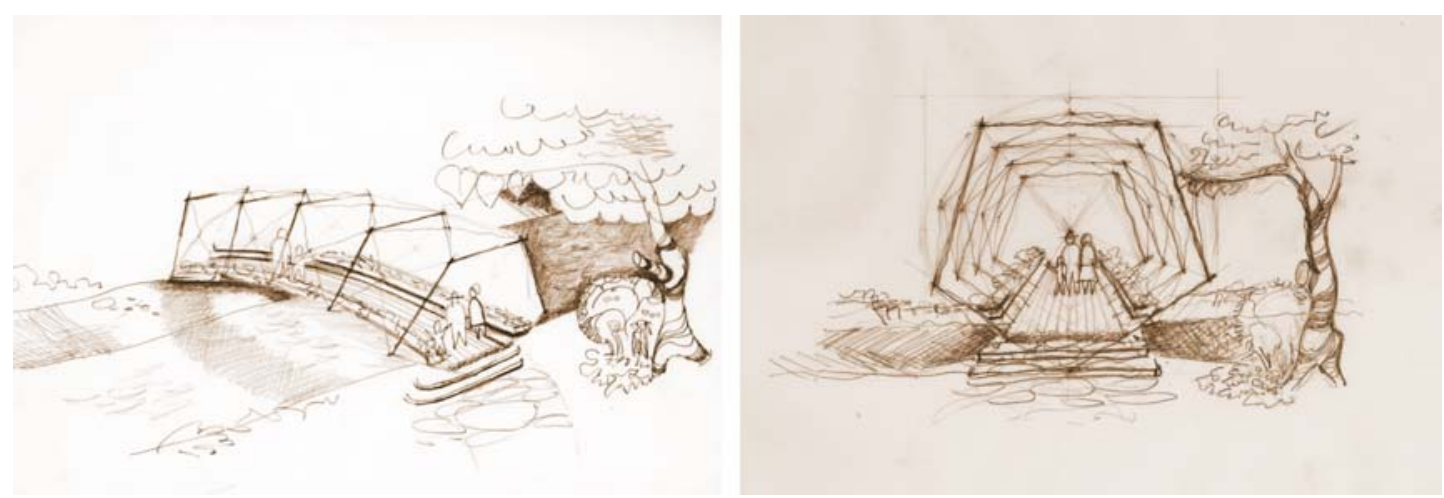

Figure 15 Bridge design applying the deployable pentagonal antiprismatic system [5] 


\section{Coilable mast}

The coilable mast system invented by Mauch [50] is originated from the idea that any elastic rod can be pushed to a helical shape [51] (cited by [2]). His lattice column is deployed through compressing the longitudinal elastic bars (called longerons) into a helical deformed shape. In the deployed configuration the stiffness is reached by bracings bars (battens) perpendicular to the longerons, and diagonal prestressed cables (Fig. 16). When releasing the coiled structure it dynamically deploys due to the accumulated energy in the folded system.
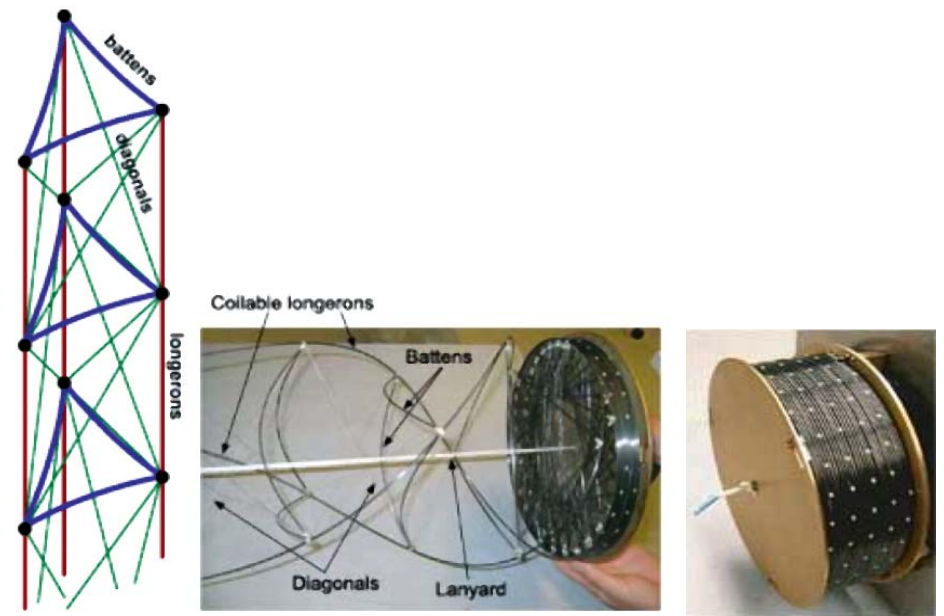

Figure 16 Coilable mast by the Jet Propulsion Laboratory

\section{Self-deployment with special joint configurations}

Accumulated energy can come from special joint configuration too. This is the case with the deployable structure presented by Fanning and Holloway [52] where the deployment is due to a spring embedded at the node, forcing the joint to rotate and consequently to deploy when releasing the structure.
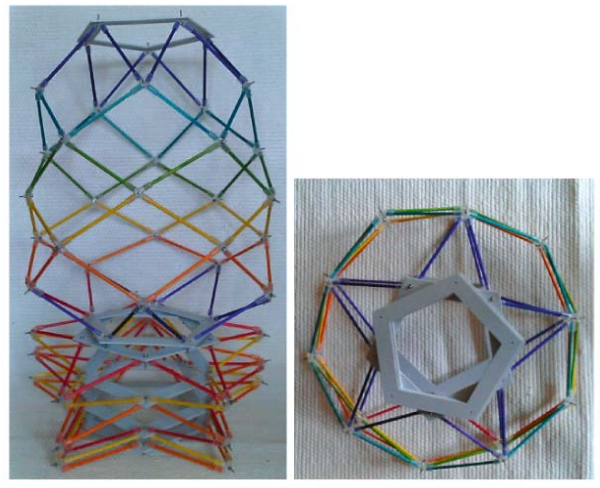

Figure 17 Physical model of antiprismatic pop-up mast by the authors (deployed and packed configurations) [5] 
A novel type of self-deploying expandable tube was derived from the antiprismatic structure presented in Chapter 2.2. (shown in Fig. 9.) Eliminating the rigid polygons of the structure and applying elastic highly flexible joints that have to undergo large rotational deformations, a popup system is created that can be packed in plane (Fig. 17).

\section{SUMMARY}

In order to give a general survey, several deployable lattice designs were presented, that offer possibilities in architectural applications. Deployable structures can be created from mechanisms that can be controlled by pneumatic systems, cables or other actuators. Tensegrity structures, which are in the first place applied for sculptures have the intrinsic possibility to be deployable. The main advantage of tensegrities is the simplicity of joints as only tendons are connected to struts in accordance with the canonical definition. However, the analysis of deployable tensegrities is extremely cumbersome; it involves highly nonlinear analysis due to prestressed cables with nonsmooth, unilateral constitutive model, finite displacement simulation, contact problems for eliminating intersection of elements during deployment etc.

A large number of deployable structures use pantographic systems. Though the scissor joint of the basic element is easy to construct, the further connection of the SLEs requires spherical joints. Furthermore, the simplest scissor joint construction evolves often non negligible friction effects. Pantographic systems can be combined with sliding joints, too, like some cable and strut systems.

If the mechanism is such that the degree of freedom of motion is only one, the control of the structure can be simple and fast. However, there is a trend to apply transformable structures not only to create a fast constructional method but to design morph shaping architectural structures to adapt the building to changing occupant demands and for reducing environmental impact and for a better energy efficiency. Morph-shaping structures are currently one of the most prospective research topic of civil engineering structures. In fact, simple control of such systems is of high complexity, a highly distributive, intelligent control and sensor system can completely transform the today's urban architecture to a dynamic, more economical, and less intrusive environment.

The second category of deployable structures is the snap-through type structures. These systems are either self-deploying or self-locking systems, depending on the stability of the equilibrium state in the packed configuration. While the self-locking system is stable and stressfree in the packed configuration, the pop-up structures are in an unstable equilibrium state in their compact configuration, corresponding to the maximum of the stored strain energy. This state enables the structure to dynamically self-deploy. The main advantage of these structures is that no additional stabilizing element is needed that makes the construction extremely rapid. The major problems of self-locking and self-deploying systems is that omitting additional poststiffening often results in the lack of sufficient structural stiffness or the occurrence of massive deploying stresses during deployment/packing. Furthermore, the complexity of analysis is of high order due to the non negligible inertial effects occurring at snapping of the structure.

In this article it was shown that already a great deal of different transformable systems are invented, however, it would be inadequate to say that application possibilities in the field of civil engineering and architecture are already well established. The difficulty arouses from either the 
need of complex and cumbersome fabrication process (in the case of a larger scale structure) or in other cases the problem comes from the necessary highly nonlinear analysis of these flexible systems. The (very briefly) presented examples show that even the analysis of a single basic element can be cumbersome and may require profound understanding of nonlinear behavior. When analyzing the interconnected single elements together the complexity of the simulation become even of higher order. Possibly the biggest challenge is that unlikely to conventional structures the inertial effects during the transformation process, as well as the uncertain energy absorptions, are of high importance in general and consequently cannot be ignored in the analysis. The architectural design work for individual applications, the elaborating calculation process and the lack of standardizable procedure for dynamical calculation and the necessary calculation costs will possibly keep the artists, the architects and the engineers busy for a while. At least the ones that have a hunger to feed their mind. Eventually and hopefully, combining transformable structures with a highly distributed control system which is already available in today's technology an intelligent responsive architecture will be born.

\section{REFERENCES}

[1] Gantes C.J., Deployable Structures: Analysis and Design, WIT Press, Southampton, Boston, 2001

[2] Pellegrino S., Deployable Structures, Springer-Verlag Wien, New York, 2001

[3] Wada B.K., Fanson J.L., Garba J.A., Chen G.S., Adaptive structures to meet future requirements for large precision structures, Proceeding of the International Conference on Spacecraft Structure and Mechanical Testing, Noordwijk, Netherlands, 1988, 121-126.

[4] Vu K.K, Tran T.C., Liew J.Y.R., Krishnapillai A., Deployable Tension-Strut Structures: From Concept to Implementation, Journal of Constructional Steel Research Volume 62, (2006), 195-209.

[5] Friedman N, Ibrahimbegovic A., Farkas Gy., Investigation of highly flexible, deployable structures : review, modelling, control, experiments and application; Phd Dissertation submitted to ENS de Cachan and to BME, tel-00675481, version 1, http://tel.archives-ouvertes.fr/tel-00675481, oai:tel.archives-ouvertes.fr:tel-00675481

[6] Iвrahimbegovic A., On the geometrically exact Formulation of Structural Mechanics and Its Applications to Dynamics, Control and Optimization, Comptes Rendus de l'Academie des Sciences, Part II : Mécanique, Volume. 331 (2003), 383-394.

[7] Ibrahimbegovic, A., Schiehlen W., Computational Techniques and Applications in Nonlinear Dynamics of Structures and Multibody System, Proceedings EUROMECH-427, Cachan, September 24-27, 2001

[8] Ibrahimbegovic A., Taylor R. L., Nonlinear Dynamics of Flexible Multibody Systems, Computers and Structures, Volume 81, (2003), 1113-1132.

[9] Gant C., Conceptual Design of Structures: Proc. of the international symposium, University of Stuttgart,, Oct. 7-11 Stuttgart, IASS, IABSE (1996), 222-229.

[10] Ibrahimbegovic A., Mamouri S., Taylor R.L., Chen A., Finite Element Method in Dynamics of Flexible Multibody Systems: Modeling of Holonomic Constraints and Energy-Conserving Integration Schemes, Multibody System Dynamics, Volume 4,(1996), 195-223.

[11] Ibrahimbegovic A., Taylor R.L., On the role of frame-invariance of structural mechanics models at finite rotations, Computer Methods in Applied Mechanics and Engineering, Volume 191, (2002), 5159-5176.

[12] Ibrahimbegovic A., Al Mikdad, Quadratically Convergent Direct Calculation of Critical Points for 3D Structures Undergoing Finite Rotations, Computer Methods in Applied Mechanics and Engineering, Volume 189, (2000), No 1. 107-120.

[13] Snelson, K., Forces made visible, Hudson Hills Press LLC, 2009

[14] Motro R., Tensegrity: Structural Systems for the future, Butterworth-Heinemann; 2006

[15] Burkhardt R.W., A Practical Guide to Tensegrity Design, Cambridge, Massachusetts: Software Services, 1994

[16] Hugh A., An Introduction to Tensegrity, Berkeley, California: University of California Press, 1976 
[17] Motro R., Bouderbala M., Lesaux C., Cévaer F. Foldable tensegrities, Deployable Structures, Springer-Verlag Wien, New York, (2001), 199-238.

[18] http://www.lt.ar.tum.de/forschung-am-lt/bewegliche-tragwerke/

[19] Rhode-Barbarigos L.G.A., Bel Hadj Ali N., Motro R. and Smith I.F C.. Design Aspects of a Deployable Tensegrity-Hollow-rope Footbridge, in International Journal of Space Structures, Volume 27, (2012), No. 2, 81-96.

[20] Атакe K., Diagonal and Variable Frame Structures, Symmetry Culture and Science Extended Abstracts 1, ISIS Symmetry, Volume 1 (1995), 53-56.

[21] Pinero E.P., Project for a mobile theatre, Architectural Design, Volume 12, (1961), 154-155.

[22] Escrig F., Brebbia C. Mobile and Rapidly Assembled Structures, II, First ed. Computational Mechanics, Southhampton, UK, (1996)

[23] Escrig F., Valcarcel J.P., SAnchez J.S., Las Cubiertas Desplogables de Malla Cuadrangular, Boletín Academico de Universidad de Coruña, Escuela Téchnica Superior de Arquitectura,-No20, 1996

[24] Pellegrino S., Kwan A. S. K., You Z., Active and passive cable elements in deployable masts, Int. J. Space Structures, Volume 8, (1993), No. 1-2, 29-40.

[25] Kawaguchi M., Авe M., On some characteristics of pantadome system, IASS 2002: Lightweight Structures in Civil Engineering, proceedings of the international symposium, Warsaw, Poland, 24-28 June, Micro-Publisher Jan B. Obrebski, (2002), 50-57.

[26] Onoda J., Fu D.-Y., Minesugi K., Two-Dimensional Deployable Hexapod Truss, Journal of Spacecraft and Rockets, Volume. 33, (1996), No.3, 416-421.

[27] LiEw J.Y.R, LeE B.H., WANG B.B., Innovative use of star prism (SP) and dipyramid (DP) for spatial structures, Journal of Constructional Steel Research, Volume 59, (2003), Issue 3, 335 - 357.

[28] Krishnapillai A., Liew J.Y.R., Vu, K. K., Deploy \& Stabilize Spatial Structures, Extended Proc. of the IASS 2004 Symposium, Montpellier, France (2004)

[29] Tran T.C., Liew R.J.Y., Butterfly structure for spatial enclosures, Journal of the International Association for Shell and Spatial Structures: IASS, Volume 47, (2006), No. 3

[30] Hoberman C., Reversibly expandable doubly-curved truss structure, US Patent No 4,942,700, (1990)

[31] Hoberman C., Radial expansion/retraction truss structures, US Patent No 5,024,031. (1991)

[32] You Z., Pellegrino S. Foldable bar structures, Int J. Solids Struct, Volume 34 (1997), No 15, 1825-1847 .

[33] Whitehead I., Steel mesh and engineering wizardry unfold into a dome, Architectural Record, 2000.oct. (2000), 79-80.

[34] Kovács, F., Mozgatható Forgásszimmetrikus és Gömbi Kupolaszerkezetek, Építés-Épitészettudomány Volume 31, (2004), No 3-4, 205-222.

[35] Kovács F., Symmetry-adapted mobility and stress analysis of spherical and polyhedral generalized bar-and-joint structures, PhD dissertation submitted to the BME, Budapest, Hungary (2004)

[36] Kокаwa T., Structural idea of retractable loop-dome, Journal of the International Association for Shell and Spatial Structures (IASS), Volume 41, (2000), No. 2, 111-116.

[37] Kassabian P. E., Investigation into a type of deployable roof structure, Final year project, University of Cambridge, Engineering Department, 1997

[38] Kassabian P.E., You Z., Pellegrino S., Retractable roof structures, Proceedings of Institution of Civil Engineers Structures and Buildings, Volume 134 (1999), No1, 45-56.

[39] Tibert, G., Deployable Tensegrity Structures for Space Applications, Doctoral Thesis for the Royal Institute of Techn. Dpt. of Mechanics, (2002)

[40] Herder, A., Synchronised Activation of Single-curved Tensegrity Grids for Responsive Architecture, http://www. arnoudherder.nl/Figures/Tensegrity/Synchronised 20 Activation 20 of 20 Single-curved 20 Tensegrity 20 Grids 20 for 20 Responsive 20 Architecture.pdf (2008)

[41] Sofla A.Y.N, Elzey D.M., WAdley, H.N.G. Shape morphing hinged truss structures, Smart Materials and Structures 18 doi:10.1088/0964-1726/18/6/065012 (2009) 
[42] Hegedüs I.: Branching of Equilibrium Paths in a Deployable Column, International Journal of Space Structures, Special Issue, vol 8, 1993, 119-125

[43] Clarke R.C., The kinematics of a novel deployable space structure system, Proceedings of the 3rd, H. Nooshin: International Conference on Space Structures, University of Surrey, Guildford, UK, London, (1984), 820-822.

[44] Zeigler T.R., Collapsible self-supporting structure. Us Patent No.3,968,808, (1976)

[45] Zeigler T. R., Collapsible self-supporting structures, Us Patent No.4,026,313 (1977)

[46] Krishnapillai A., Zalewski W. P., The design of deployable structures - Kinematic design, Unpublished Research Report, Departent of Architecture, MIT, Cambridge, Massachussets, USA, October (1985)

[47] Krishnapillai A., Crawley E., Deployable structures - Kinematic design, Unpublished Research Report, Departent of Aerospace Engineering, MIT, Cambridge, Massachussets, USA, October, 1986

[48] Mikulas M.M.Jr., Wada B.K., Farhat C., Thorwald G., Withnell P., Initially Deformed Truss Geometry for Improving the Adaptivity Performance of Truss Structures, Third International Conference on Adaptive Structures, Wada B.K., Natori M, Breitback E, Nov 9-11, San Diego, California, USA, (1992), 305-319.

[49] Friedman $\mathbf{N}$ et al., On the snap-back behavior of a self-deploying antiprismatic column during packing. Eng Struct (2013), http://dx.doi.org/10.1016/j.engstruct.2012.12.035

[50] Webb J.E., Mauch H.R., Deployable Latice Column, USA Patent No. 3486279 (1969)

[51] Love A.E.H., A treatise on the mathematical theory of elasticity, Fourth Edition, Dover Publications, New York, (1944)

[52] Fanning P.,Holloway L., The Deployment Analysis of a Large Space Antenna, International Journal of Space Structures, Volume 8, (1993), No. 3, 209-220. 